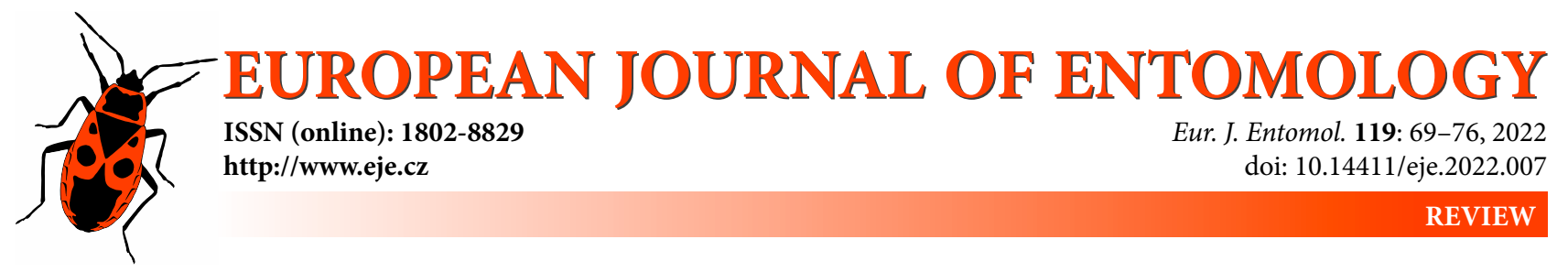

\title{
Time measurement in insect photoperiodism: The role of photophase duration and light intensity*
}

\author{
DAVID SAUNDERS \\ 21 Leadervale Road, Edinburgh EH16 6PB, Scotland, United Kingdom; e-mail: david59.saunders@mypostoffice.co.uk
}

Keywords. Diapause, Pyrrhocoris apterus, day- or nightlength measurement

\begin{abstract}
The initial stages of diapause induction - as summer gives way to autumn - involve a process of time measurement in which the duration of daylength (or nightlength) is determined by a photoperiodic 'clock' based upon the circadian system. In many insects so far examined, a photophase of sufficient duration and illuminance resets a photoperiodic oscillator to a constant phase equivalent to the beginning of the 'subjective night' (Circadian time, CT $12 \mathrm{~h}$ ) whereupon it proceeds to measure nightlength in a clock of the external coincidence type. A possible exception may be found in the linden bug, Pyrrhocoris apterus, which - in laboratory studies using relatively low light intensity - suggests that daylength is measured rather than the night. Earlier studies of photoperiodic responses (pupal diapause induction) in the flesh fly Sarcophaga argyrostoma, however, showed that 'weak' or short light pulses giving rise to Type 1 phase response curves could be converted by increasing light intensity to Type 0 responses that phase set the oscillation to the beginning of the subjective night (CT $12 \mathrm{~h}$ ) whereupon it could begin to measure the night. Based upon these data it is therefore suggested that the photoperiodic clock in $P$. apterus might also measure nightlength if the bugs were exposed to photophases of higher irradiance simulating daytime exposure to the light intensity experienced by these diurnally active insects in their natural environment.
\end{abstract}

\section{INTRODUCTION}

Marcovitch $(1923,1924)$ - no doubt encouraged by Garner \& Allard's (1920) seminal observations with plants - provided the first experimental evidence for photoperiodism in insects, specifically for the regulation of seasonal morphs in aphids. Demonstration of the photoperiodic control of diapause induction followed with the works of Kogure (1933) and Sabrosky et al. (1933) on the commercial silkworm and grouse locusts, respectively.

Early investigations attempted to establish the approximate light intensity thresholds for various aspects of the photoperiodic reaction. For example, the threshold for larvae of the Oriental fruit moth, Grapholita molesta within young apples was about 10 to $30 \mathrm{~lx}$ (Dickson, 1949) whereas that for exposed larvae of the noctuid Lacanobia (Diataraxia) oleracea was also about 10 lx (Way \& Hopkins, 1950). Codling moth (Cydia pomonella) larvae outside the fruit appeared to be much more sensitive (quoted as about $0.02 \mathrm{~W} \mathrm{~m}^{-2}$ ) for diapause termination (Norris et al., 1969) and the larvae of the midge Metriocnemus knabi living within the pitcher plant Sarracenia purpurea showed a threshold sensitivity close to $0.025 \mathrm{~lx}$ (Paris \& Jenner, 1959). Although light 'intensity' in these examples was re- corded as either illuminance (lx) or irradiance $\left(\mathrm{W} \mathrm{m}^{-2}\right)$ and therefore not easily compared, these data suggested that insects varied in their sensitivity to light, often according to their ecology, some 'seeing' given light as 'dim', others 'seeing' the same light as 'bright'. These variable responses to light intensity are further examined in this review with particular emphasis on the relationship between light pulse duration and illuminance within the context of diapause induction and the nature of the photoperiodic clock.

This review is dedicated to the memory of Ivo Hodek who in his long career championed Andrewartha's (1952) concept of 'diapause development' and contributed much fundamental work on the physiology of diapause (Hodek, 1999, 2002), particularly in that of adult insects.

\section{The role of circadian rhythmicity in photoperiodic time measurement}

The central role of time measurement in the inception of diapause (= diapause induction) was rarely addressed in early studies and, when it was, it was frequently attributed to a linear, biochemical hourglass-like mechanism measuring either the duration of the day, or the night. In 1936, however, the German plant physiologist Erwin Bün-

\footnotetext{
* This paper was contributed to a virtual special issue in memory of Ivo Hodek, a long-time editor of the European Journal of Entomology, who died on June 11, 2021, shortly after his ninetieth birthday.
} 
ning (1936), working with bean seedlings, suggested that time measurement in photoperiodism was a function of the circadian system (see Saunders, 2020, 2021a) and, moreover, that the daily up-and-down movements of the seedling leaves acted as an overt manifestation of the otherwise hidden photoperiodic oscillation. Full exposure of Bünning's hypothesis, however, only occurred in 1960 at the Cold Spring Harbor Symposium on "Biological Clocks" (Chovnick, 1960) and then became a valid alternative model for photoperiodic time measurement in both plants and animals.

Colin Pittendrigh became an ardent supporter of Bünning's hypothesis, noting the similarities between photoperiodic induction and his own work on the circadian rhythm of adult emergence (eclosion) of Drosophila pseudoobscura - despite this particular species' lack of a diapausing stage in its life cycle. His interest in this possible connection led to the development of several models for the induction of insect diapause (Pittendrigh, 1966, 1972) described later in this review.

Two aspects of circadian eclosion rhythmicity were relevant in this comparison. The first was that transfer of fruit fly pupae from light into darkness initiated a rhythm of eclosion that persisted ('free ran') in subsequent darkness - a phenomenon discovered earlier by Bünning himself (Bünning, 1935; but see also Kalmus, 1935). The second was Pittendrigh's observation that when a circadian oscillation such as that controlling pupal eclosion was exposed to pulses of light, these stimuli frequently caused a phase shift, advance or delay. This meant that any analysis of photoperiodic responses should also consider such phase shifts and had to involve description of these changes in terms of phase response curves (PRCs). These phenomena subsequently formed a basis for the analysis of photoperiodic induction of diapause in insects, including the importance of light intensity.

\section{The use of phase response curves in the analysis of insect photoperiodism}

When mixed age populations of D. pseudoobscura were transferred from continuous light (LL), or from a long photophase, into continuous darkness (DD), peaks of adult eclosion were generated at intervals of about 24 $\mathrm{h}$, constituting an overt circadian rhythm. In a now classic experiment using the natural cool waters of a Rocky Mountain stream, Pittendrigh (1954) demonstrated that the endogenous period of this oscillation $(\tau \mathrm{h})$ was temperature compensated between 16 and $26^{\circ} \mathrm{C}$ (i.e. with a temperature coefficient, $\mathrm{Q}_{10}$ close to 1.0), thereby suggesting that the circadian oscillation could act as a biological 'clock'. Apart from the peaks of eclosion, however, there were no other visible markers of the underlying oscillation, although systematic probing by short light pulses provided a 'time course' of the oscillation in the form of a phase response curve, as shown in Fig. 1.

When the circadian oscillation was persisting or 'freerunning' in continuous darkness (DD) it was shown to pass through successive half-cycles corresponding to 'subjective nights' followed by 'subjective days' (i.e. phases that
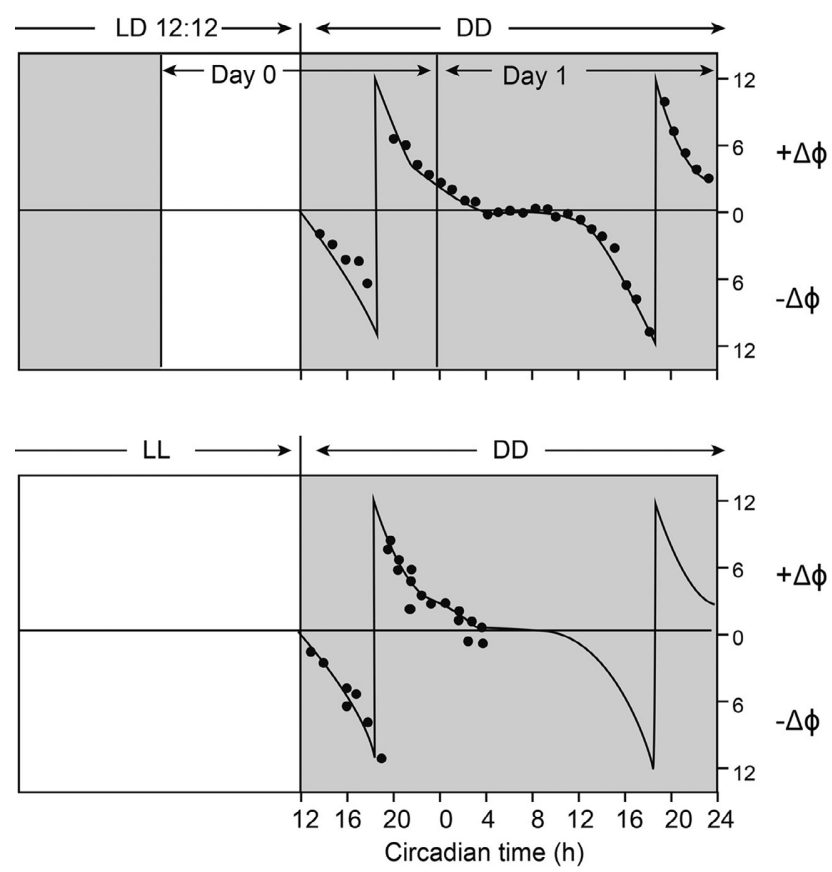

Fig. 1. The time course of the pupal eclosion oscillator in Drosophila pseudoobscura after transfer from a light cycle (12L : 12D) into constant darkness (DD) (upper panel), or from constant light (LL) into DD (lower panel). The oscillation is presented as a phase response curve (PRC) with phase delays $(-\Delta \varphi)$ in hours below the midline and phase advances $(+\Delta \varphi)$ above it; the PRC in the lower panel is the same as that in the upper. In both regimes the oscillation is set to a constant phase (Circadian time, CT $12 \mathrm{~h}$ ) equivalent to the start of the subjective night. After Pittendrigh (1966).

would be occupied by day and night if the insects were in continued light cycles) (Pittendrigh, 1966). Short light pulses (15 min of white light of about 500 lux) probing the first half of the subjective night were then found to cause increasingly great phase delays $(-\Delta \varphi)$ followed by phase advances $(+\Delta \varphi)$ in the second half of the subjective night, whilst the ensuing subjective day was largely immune to such phase changes (Fig. 1). Pittendrigh (1960), using light pulses of $12 \mathrm{~h}, 4 \mathrm{~h}$ and $1 / 2000 \mathrm{~s}$ also showed that the magnitude of the phase shifts generated depended on the 'strength' - i.e. the duration and probably also the intensity of the light pulses perturbing the rhythm.

Winfree (1970, 1980), working in the Pittendrigh laboratory, extended these studies using pulses of dim blue light of varying duration applied at intervals to an eclosion rhythm generated by transferring insects from LL into DD. When the test pulses were less than about $50 \mathrm{~s}$ in duration the resulting phase shifts were small giving rise to low 'amplitude' PRCs, whereas longer pulses caused phase shifts of up to $12 \mathrm{~h}$ to give high 'amplitude' PRCs (Fig. 2 , left-hand panels). Another way of presenting the same resetting data is to plot the resulting phase ('new phase') as a function of the phase at which the pulse was initiated ('old phase'), giving rise to phase transition curves (PTCs). With 'weak' (i.e. short or low intensity light) pulses the resultant curve has an average slope close to 1 , whereas 'stronger' (i.e. longer or more intense) pulses give rise to a curve whose average slope is closer to zero (Fig. 2, right- 

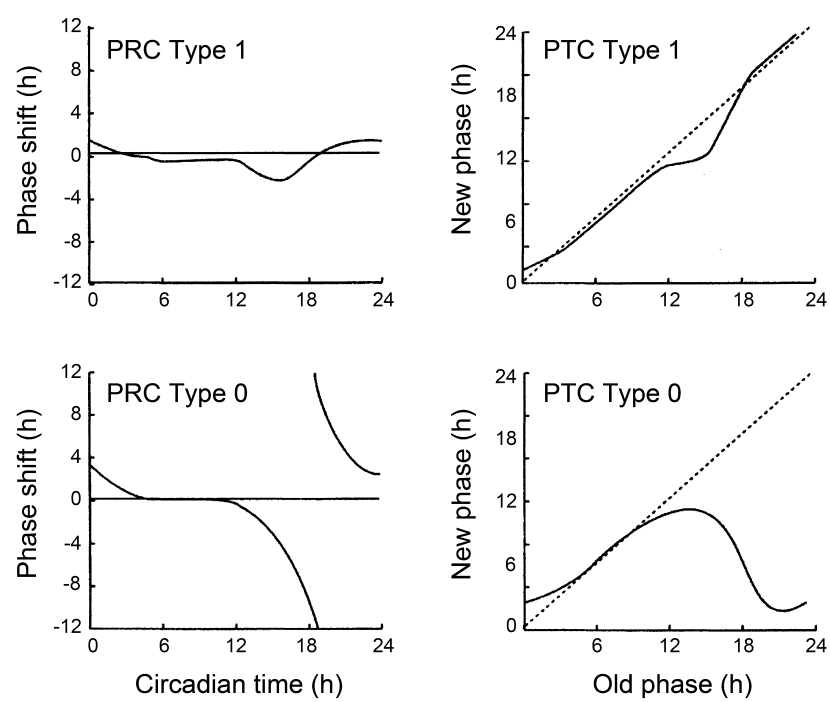

Fig. 2. Schematic representations of circadian rhythm phase response curves (PRCs, left-hand panels) or phase transition curves (PTCs, right-hand panels). In PRCs, responses to 'weak' (i.e. short or low intensity) light pulses (upper left) are small, whereas responses to 'strong' (longer or brighter) pulses (lower left) are up to $12 \mathrm{~h}$ in magnitude. In PTC plots the same data are plotted as 'new phase' (after the pulse) as a function of 'old phase' (before the pulse). With 'weak' light pulses (upper right) the PTC has a slope of 1 whereas 'strong' pulses (lower right) result in a slope approaching zero. The two types of PTC are referred to as Type 1 and Type 0 responses. Redrawn after Winfree (1970).

hand panels). With even longer or brighter pulses the PTC becomes a straight line with a slope of 0 (example shown in Fig. 3) showing that the principal time cue is now taken from light-off; the two types of response were referred to as Type 1 and Type 0 , accordingly. With Type 0 PRCs, lightoff initiates a rhythm at a phase equivalent to the beginning of the subjective night, a phase referred to as Circadian time, CT $12 \mathrm{~h}$, an observation of crucial importance in the analysis of photoperiodic time measurement (Section 4).

Winfree $(1970,1980)$ also raised cultures of $D$. pseudoobscura in LL, 'released' them into DD to generate a clear rhythm of eclosion starting at CT $12 \mathrm{~h}$, and then probed the rhythm at variable times $(\mathrm{T})$ after the LL/DD transfer with pulses (S) of dim blue light. When $\mathrm{T}$ and $\mathrm{S}$ were varied independently a $50 \mathrm{~s}$ pulse placed $6.8 \mathrm{~h}$ after the LL/DD transition (i.e. at CT $18.8 \mathrm{~h}$, close to 'subjective midnight') was found to induce a state of arrhythmicity, a phenomenon considered to represent the action of a critical pulse, 'stopping the clock' by driving the oscillation onto its 'singularity' (see also, below).

\section{Sensitivity to light}

Several pieces of evidence suggested that the eclosion rhythm in Drosophila pseudoobscura was very sensitive to light. Winfree (1974), for example, found that transfer of rhythmic populations in DD, with an inter-peak interval $(\tau)$ of about $24 \mathrm{~h}$, into continuous, very low intensity, blue light caused $\tau$ to increase to about $24.7 \mathrm{~h}$, but exposure to somewhat higher intensity caused adults to emerge at random, the two extremes being likened to 'starlight' and 'moonlight', respectively. Some other aspects of the rhythm also

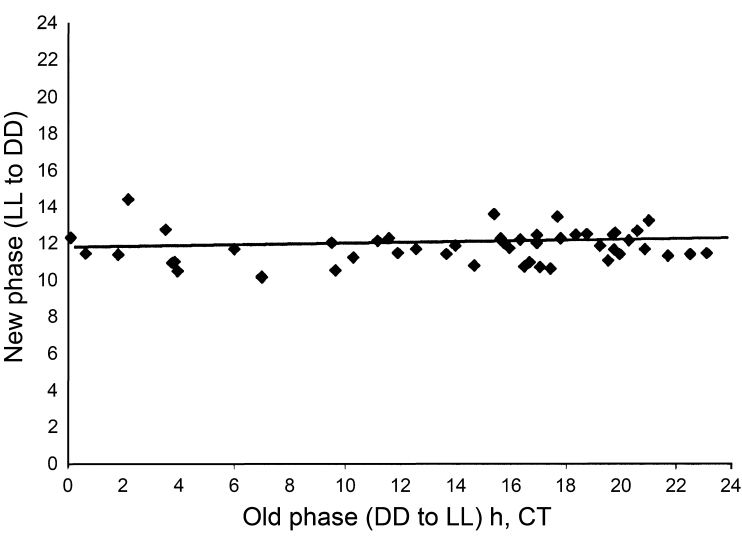

Fig. 3. Type 0 PTC showing resetting the locomotor activity rhythm of the blow fly Calliphora vicina after an extended period of light into darkness showing that the oscillation is completely phase-set to CT $12 \mathrm{~h}$ (the beginning of the subjective night) at light off, regardless of the circadian phase at light-on. Redrawn, in part, from Saunders \& Cymborowski, 2008).

showed that an increase in irradiance - although sometimes over a limited range - was, like pulse duration, correlated with the magnitude of response (Engelmann, 1969; Chandrashekaran \& Loher, 1969; Chandrashekaran \& Engelmann, 1973) showing that both the duration of the light pulse and its intensity act in a similar fashion upon the system. Other phenomena, such as placement of the $D$. pseudoobscura rhythm on its 'singularity' (Chandrashekaran \& Engelmann, 1976) showed that reciprocity between irradiance and duration of the critical pulse was vast, ranging from very short pulses (0.04 s) of very bright light $\left(12,500 \mathrm{~W} \mathrm{~m}^{-2}\right)$ to very long pulses $(13.9 \mathrm{~h})$ of dim light at $0.01 \mathrm{~W} \mathrm{~m}^{-2}$. It is possible, therefore, that similar reciprocity between duration of a light pulse and its intensity occurs in other aspects of circadian and photoperiodic responses; these will be discussed later in this review (Section 5).

\section{Models for the photoperiodic clock}

Models for photoperiodic induction began with Bünning $(1936,1960)$ who proposed that light had two roles: entraining (phase setting) the endogenous circadian rhythm and activating a 'long-day' response by illuminating a particular light-sensitive phase in the night. This model was further developed by Pittendrigh (1966) - based mainly on his work on the behaviour of the eclosion rhythm of $D$. pseudoobscura outlined above - and referred to as external coincidence (Pittendrigh, 1972). Like Bünning's original version, it was considered to comprise a single oscillator, but (in insects) reset by light pulses in excess of about 12 $\mathrm{h}$ to a narrow range of phases close to CT 12 (see above), with the light-sensitive (or 'photoinducible') phase $\left(\varphi_{\mathrm{i}}\right)$ occurring late in the night, at the end of the "critical nightlength' (Fig. 4). External coincidence, as formulated by Pittendrigh (1966), accounted for the principal characteristic of a 'long-day' insect's photoperiodic response curve (PPRC) - namely the critical daylength or nightlength separating the diapause and nondiapause regulatory pathways - but did not explain the fall in diapause incidence under 'ultrashort' photophases shown in typical examples of in- 


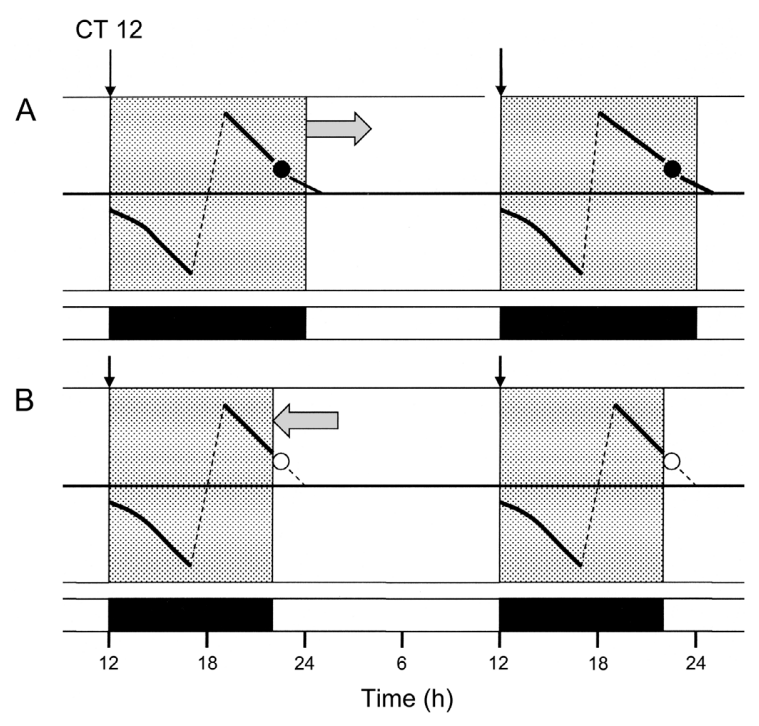

Fig. 4. The external coincidence model for the insect photoperiodic clock. A - as autumn approaches and nights lengthen, the dawn transition is delayed (horizontal arrow) with respect to the circadian time scale, $\varphi_{i}$ (the photoinducible phase) begins to fall in the dark and diapause supervenes. $\mathrm{B}-$ in the summer, the dawn transition occurs earlier (arrow) with respect to the circadian time scale, $\varphi_{i}$ is illuminated and nondiapause development proceeds. Closed circles show photoinducible phase in the dark; open circles show the same phase in the light. Redrawn after Pittendrigh (1966).

sect photoperiodic responses (Fig. 5). Reasons for this fall in diapause incidence will be addressed in Section 5 below.

External coincidence (Fig. 4 upper panel) suggests that as autumnal nights lengthen, $\varphi_{\mathrm{i}}$ begins to fall in the dark and diapause supervenes. Fig. 4 (lower panel) also suggests, however, that as days lengthen, dawn light occurs earlier, eventually to coincide with $\varphi_{\mathrm{i}}$ and thereby leading to nondiapause development. Such photoperiodic termination of diapause occurs in some species - perhaps those in southerly environments with a warm spring - but is not typical of those populations inhabiting localities further to the north. For example, working with the linden bug, Pyrrhocoris apterus, in central Europe (Bohemia), Hodek (1971) transferred overwintering bugs from field populations, at intervals during the winter, to the laboratory and showed that diapause had ended (i.e. 'diapause development' was completed) by the end of December and the insects then remained in a cold-induced reproductive quiescence until April when temperatures began to rise. Photoperiodically regulated diapause in P. apterus (and many other mid-latitude species) was therefore an autumnal phenomenon preventing maladaptive reproductive activity during the autumn and early winter and not necessarily associated with processes of reactivation.

External coincidence has proved useful for analysis of photoperiodic induction in several species of Diptera (Section 5) including some high-latitude drosophilids which, however, may incorporate heavily dampened oscillators (Vaze \& Helfrich-Förster, 2016; Lankinen et al., 2021). This model, however, cannot be regarded as universal, other possible models being appropriate elsewhere (Vaz Nunes \& Saunders, 1999).

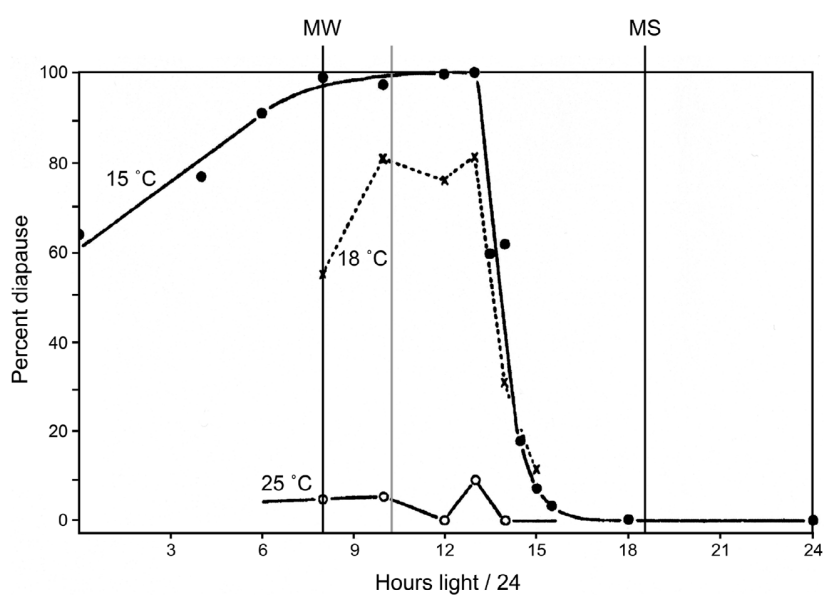

Fig. 5. The photoperiodic response curve for larvae of the flesh fly, Sarcophaga argyrostoma, showing the critical photoperiod between long and short daylengths at about $14.5 \mathrm{~h} / 24$, the effect of temperature on the incidence of resulting pupal diapause under short days, and the fall in diapause under very short daylengths. The bold vertical lines show the approximate midwinter (MW) and midsummer (MS) photoperiods at $55^{\circ} \mathrm{N}$, the origin of the fly strain. The faint vertical line marks the daylengths which only occur during the winter when the flies at cool temperature are already in diapause. Redrawn from Saunders (1971).

\section{Application of the external coincidence model to photoperiod-positive species}

Intra-uterine embryos and feeding larvae of the flesh fly Sarcophaga argyrostoma exposed to the long days of summer develop without further arrest, but those exposed to autumnal short days (long nights) enter diapause in the pupal instar (Saunders, 1971) (Fig. 5). Exposure of the photoperiod-sensitive stages to Nanda-Hamner experiments (Nanda \& Hamner, 1958; Saunders, 1973, 2021a; see also Teets \& Meuti, 2021) also showed a high incidence of diapause in light-dark cycles whose periods were 24,48 or $72 \mathrm{~h}$, but a low incidence of diapause when periods were 36 or $60 \mathrm{~h}$, indicating the circadian basis of photoperiodic time measurement in S. argyrostoma (Fig. 6), the declining amplitude of the high diapause peaks probably indicating a slowly dampening oscillation as development proceeds. Long day, non-diapausing individuals also showed an endogenous circadian rhythm of adult emergence with peaks of eclosion close to dawn. This species, therefore, presented an opportunity of analysing photoperiodic induction, using the overt rhythm of pupal eclosion as an indicator of phase ('hands of the clock') of the otherwise covert photoperiodic system in one and the same species (Saunders, 1978).

After transfer of first instar larvae of $S$. argyrostoma from LL to DD - which synchronised them to a constant phase at $\mathrm{CT} 12 \mathrm{~h}$ - cultures were exposed to pulses of white light $\left(240 \mu \mathrm{W} \mathrm{cm}{ }^{-2}\right)$ of between 1 and $20 \mathrm{~h}$ duration, scanning the following hours of darkness to give a 'family' of PRCs (Fig. 7). The same data plotted as phase transition curves (PTCs) (see Saunders, 1978) showed that 1 to $4 \mathrm{~h}$ pulses gave rise to 'weak' or Type 1 PTCs, whereas pulses of $5 \mathrm{~h}$ or more gave rise to 'stronger' or Type 0 responses whose average slope was parallel to the end of the resetting pulse. Longer light pulses thus reset the oscillation to CT $12 \mathrm{~h}$ and 


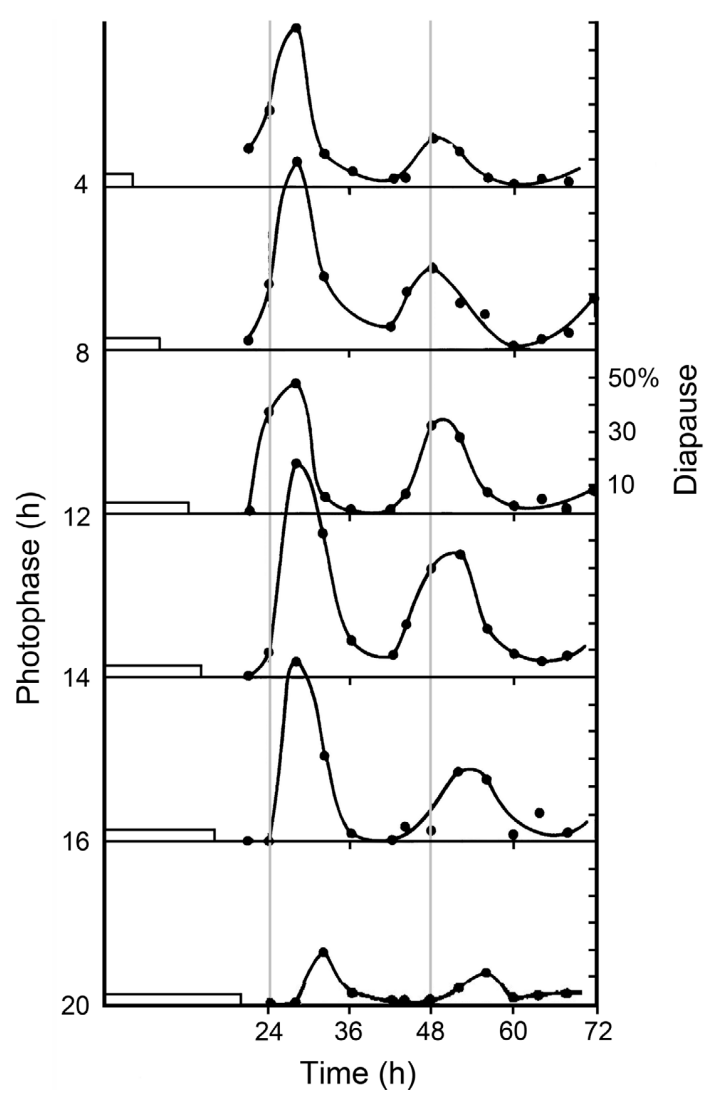

Fig. 6. Results of Nanda-Hamner experiments (see text for details) in S. argyrostoma. Horizontal bars on the left show the photophases ( $L=4$ to $20 \mathrm{~h}$ ) in each cycle. Plotted points show pupal diapause incidence after each cycle produced by $L+$ variable darkness (D) to give cycle lengths from 18 to $72 \mathrm{~h}$, repeated throughout the larval sensitive period. Pupal diapause incidence shows peaks of diapause at about $24 \mathrm{~h}$ intervals as cycle length is extended indicating circadian involvement in the photoperiodic clock. A delay in these peaks in relation to light-off following photophases longer than about $12 \mathrm{~h}$ indicates that nightlength measurement begins at the end of the photophase (at circadian time, $\mathrm{Ct} 12 \mathrm{~h}$ ). The decline in peak diapause amplitude is attributed to the dampening of the photoperiodic oscillator. Redrawn from Saunders (1973).

the following dark period was 'measured' from this dusk transition. However, since Type 1 PRCs are characterised by smaller phase shifts than Type 0 , the photoperiodic oscillation exposed to trains of weak Type 1 pulses must pass through a greater number of non-steady state or transient cycles before achieving entrainment than an oscillation exposed to a train of stronger Type 0 pulses (Saunders, 1982).

In $S$. argyrostoma, two experiments were conducted to investigate whether diapause induction was affected by the number of transient cycles experienced by the photoperiodic oscillation before it achieved steady-state (Saunders, 1982). In the first experiment, populations of larvae were transferred from LL to DD and then exposed to trains of light cycles containing photophases of 1 to $21 \mathrm{~h}$ with the light pulses starting either 'in phase' with the end of the first pulse already at CT $12 \mathrm{~h}$ (the beginning of the subjective night), or initially 'out of phase' with the first pulse starting at CT $12 \mathrm{~h}$. Fig. 8 shows that for all populations exposed to 'strong' pulses (10 to $21 \mathrm{~h}$ ), giving rise to Type 0 PRCs with high amplitude phase shifts, there were no

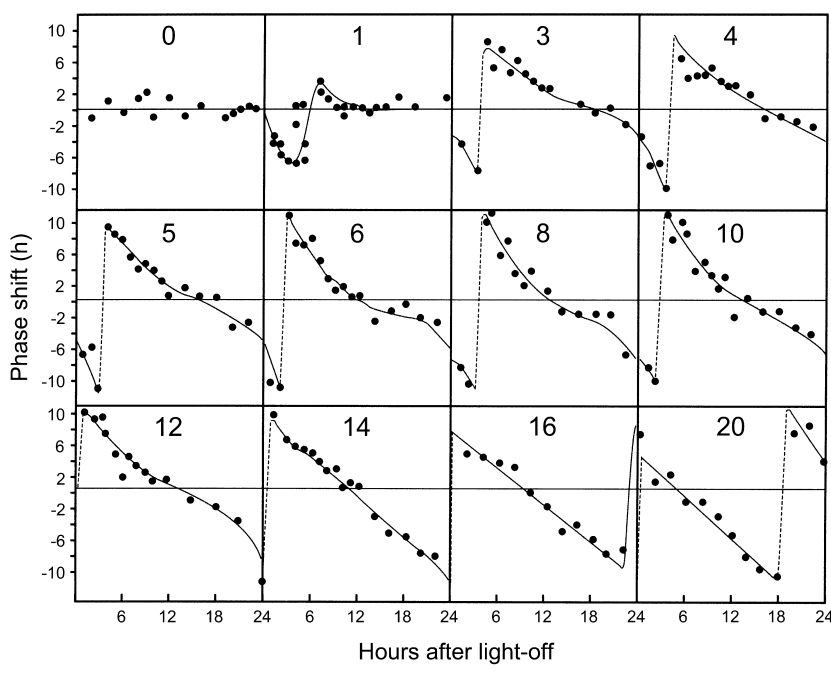

Fig. 7. Phase response curves for the adult emergence rhythm in S. argyrostoma exposed (in different panels) to light pulses starting at different phases of the oscillation. In each panel, values of phase delays $(-\Delta \varphi)$ are plotted below the mid-line, with phase advances $(+\Delta \varphi)$ above it. Very short light pulses (1 to about 3 or $4 \mathrm{~h}$ ) give rise to low amplitude Type 1 PRCs, whereas longer pulses give rise to high amplitude Type 0 PRCs. Redrawn from Saunders (1978).

significant differences between groups starting at either CT $12 \mathrm{~h}$ or CT $0 / 24 \mathrm{~h}$ because entrainment was rapid with few or no transients. With 'weaker' pulses, however - particularly those less than $6 \mathrm{~h}$ in duration giving rise to Type 1 PRCs with smaller phase shifts - the in-phase groups, already close to entrainment, produced a consistently high incidence of diapause, whereas those initially out of phase and passing through a greater number of transients before achieving steady-state (Saunders, 1982) showed a reduction.

In the second experiment (Fig. 9) larval cultures were exposed to trains of light cycles containing $2,4,8$ or 12 h photophases either at an intensity of $240 \mu \mathrm{W} \mathrm{cm}$ or at a much brighter irradiance, with the expectation that increased light intensity would also increase the 'amplitude' of phase shifts, perhaps in some cases converting Type 1 PRCs to Type 0 , and thereby decreasing transient number. Results showed that increasing the irradiance of the light pulses also increased diapause incidence, particularly for cycles containing 2 and $4 \mathrm{~h}$ light pulses, providing evidence for this expectation.

The external coincidence model has therefore proved to be appropriate for S. argyrostoma, albeit with a slowly dampening photoperiodic oscillator (Saunders, 1979). It is also indicated in S. similis (Goto \& Numata, 2009; Mukai et al., 2021). In the blow fly, Calliphora vicina, this model is also appropriate (Kenny \& Saunders, 1991; Saunders, 2021b) although with a fully self-sustained oscillation. The hourglass-like photoperiodic clocks in Megoura viciae (Lees, 1965, 1973) and the high-latitude Drosophila ezoana (Vaze \& Helfrich-Förster, 2016) and D. montana (Lankinen et al., 2021) resemble that in S. argyrostoma in most respects except that Nanda-Hamner experiments with these species fail to reveal peaks and troughs of diapause in extended periods of darkness that indicate clear circadian 


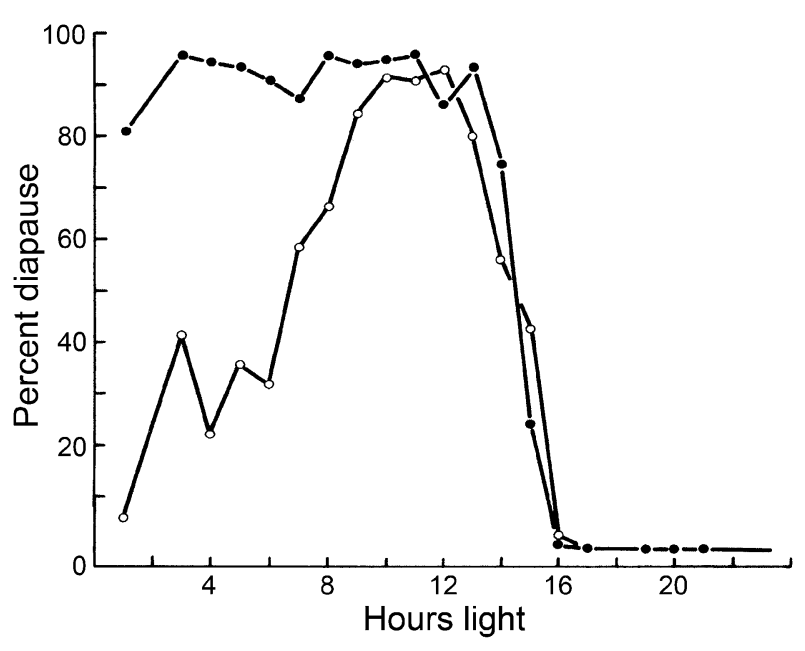

Fig. 8. Photoperiodic response curves for larvae of the flesh fly, $S$. argyrostoma exposed to trains of light pulses (1 to $21 \mathrm{~h}$ in duration) starting (closed circles) initially in-phase with their final steadystate (i.e. ending at Ct $12 \mathrm{~h}$ ) or initially out-of-phase (open circles) (i.e. starting at Ct $12 \mathrm{~h}$ ). With strong pulses (10 to $21 \mathrm{~h}$ ) which produce Type 0 PRCs with large phase shifts there were no differences between the two groups. With shorter pulses, however, particularly those of $6 \mathrm{~h}$ or less that produce Type 1 PRCs with small phase shifts, those cultures starting already in-phase with their final steady-state result in a consistently high incidence of diapause, whereas those starting out-of-phase result in a lowered incidence of diapause probably because they undergo a series of non-steady state or transient cycles before they achieve entrainment. Redrawn in part from Saunders (1982).

involvement in time measurement. The otherwise close similarity, however, suggests that nightlength in these examples is measured by a clock of the external coincidence type, but which then undergoes rapid dampening to give hourglass-like properties.

\section{Day- or nightlength measurement?}

Independent variation of the light (L) and the dark (D) components of the daily light cycle has frequently been used in attempts to ascertain their relative importance in photoperiodic time measurement: in the majority of cases the duration of D seems to be more important than L, suggesting that the photoperiodic clock most frequently measures nightlength (Saunders, 2013). One exception to this may be that of the linden bug, Pyrrhocoris apterus (Saunders, 1987) - a long-time favourite in the Hodek laboratory, now adorning the European Journal of Entomology's masthead. Using an irradiance of about $240 \mu \mathrm{W} \mathrm{cm}$ cm $^{-2}$ bugs were exposed to light cycles with $\mathrm{L}$ held constant (at 12, 15,16 or $17 \mathrm{~h}$ in different subsets) and D varied, or with D held constant (at 7, 8, 9 or $12 \mathrm{~h}$ in different subsets) and $\mathrm{L}$ varied. In each experiment the incidence of diapause was compared with the critical daylength (or nightlength) obtained from 24-h cycles (Saunders, 1983). The results (Fig. 10) showed that variation of $L$ whilst holding $D$ constant more closely resembled the natural photoperiodic response curve, suggesting that measurement of daylength was more important than that of darkness. That conclusion, however, may only be appropriate for the light intensity $(240 \mu \mathrm{W}$ c $\mathrm{m}^{-2}$ ) used in this particular investigation; use of higher ir-

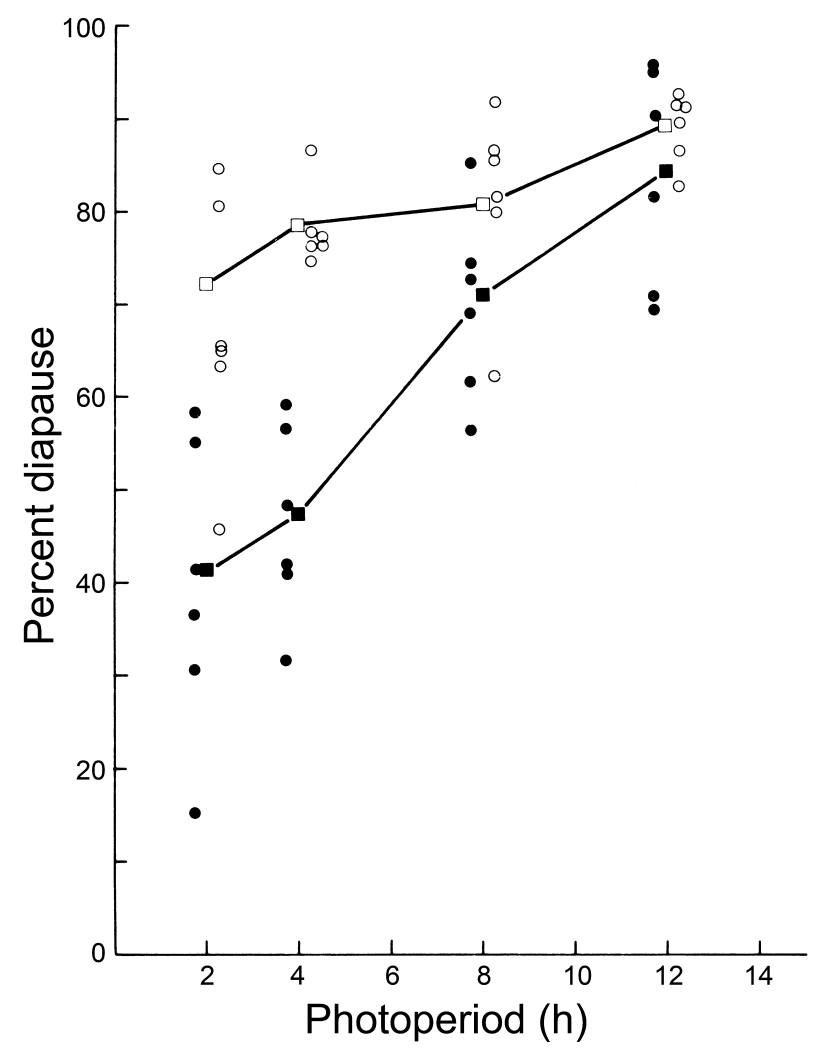

Fig. 9. Pupal diapause in $S$. argyrostoma exposed as larvae to light cycles (2L:22D, 4L:20D, 8L: 16D or 12L:12D) at two light intensities, one 'dim' (about $240 \mu \mathrm{W} \mathrm{cm}{ }^{-2}$ ) (closed circles, 5 to 6 replicate experiments) or at much 'brighter' light (about 16,000 $\mu \mathrm{W}$ $\mathrm{cm}^{-2}$ ) (open circles, 5 to 6 replicate experiments). Closed and open squares are the mean values for each treatment. Increasing the irradiance of the light pulses increases the incidence of pupal diapause, particularly for the shorter (2 and $4 \mathrm{~h}$ ) pulses, an increase probably engendered by a change in PRCs from Type 1 to Type 0 at higher light intensity. From Saunders (1982).

radiance may have produced a somewhat different conclusion (see Section 7 below).

\section{Discussion and future direction}

In laboratory experiments conducted at an irradiance of about $240 \mu \mathrm{W} \mathrm{cm}{ }^{-2}$ (Saunders, 1987), photoperiodic time measurement in P. apterus appeared to begin at light-on and to measure the duration of daylength. Furthermore, nymphal locomotor activity and the cyclical expression of the circadian clock genes period and cycle in nymphal heads showed a similar rhythmic pattern at $50 \mu \mathrm{W} \mathrm{cm}$ (Kotwica-Rolinska et al., 2017) - an irradiance considerably lower than that quoted above. RNAi knockdown of the clock genes Clock and cycle during nymphal development led to reproductive arrest, expression of the storage protein hexamerin in the fat body but reduced vitellogenin in the egg follicles. Taken together, these studies point to a photoperiodic mechanism based on the circadian system but one that 'measures' daylength rather than nightlength.

However, under field conditions, P. apterus is a diurnal species, frequently active in bright sunshine. In late summer and autumn - when photoperiodic induction of diapause occurs - light intensity may be several orders of magnitude greater than that provided in the laboratory studies 


\section{L constant}
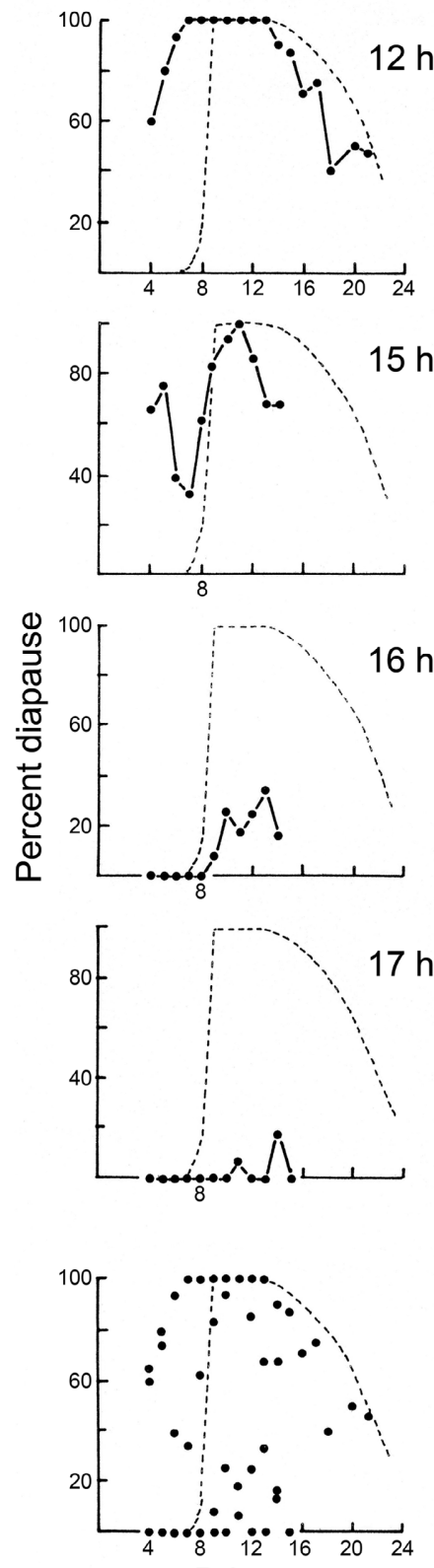

D hours

\section{D constant}
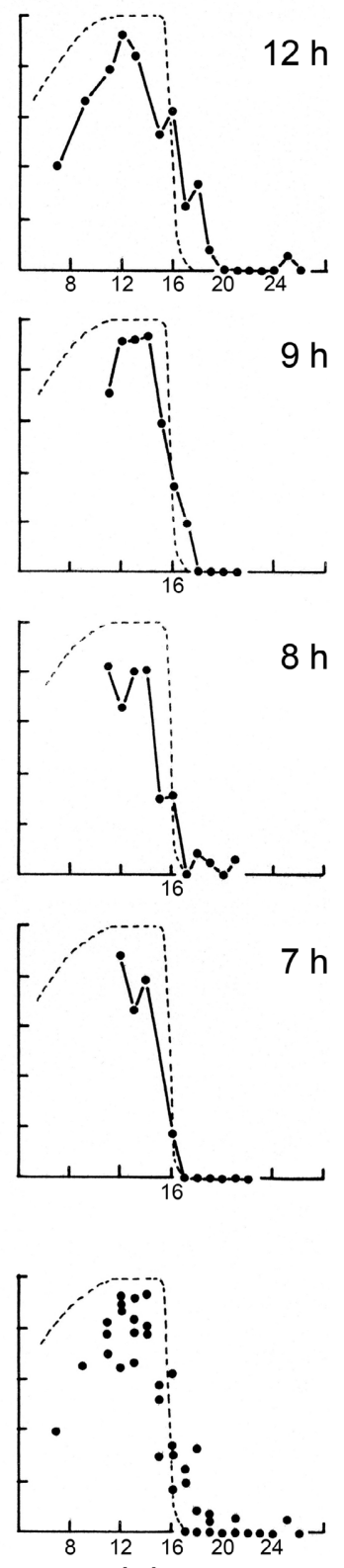

L hours
Fig. 10. Incidence of reproductive (ovarian) diapause in females of Pyrrhocoris apterus exposed, as nymphs, to independently varied light ( $\mathrm{L}$, at about $240 \mu \mathrm{W} \mathrm{cm} \mathrm{cm}^{-2}$ ) and dark (D) components of the daily photocycle. Left hand panels: L held constant (at 12, 15, 16 or $17 \mathrm{~h}$ ) and $\mathrm{D}$ varied. Right hand panels: $\mathrm{D}$ held constant (at 12, 9, 8 or $7 \mathrm{~h}$ ) and $\mathrm{L}$ varied. The two lower panels show data for $\mathrm{L}$-constant and D-constant combined. In each panel the dotted curve shows the photoperiodic response curve for $P$. apterus in a $24 \mathrm{~h}$ cycle. The combined data for $\mathrm{D}$ constant and $\mathrm{L}$ varied show a response closer to that in the $24 \mathrm{~h}$ cycle, suggesting that daylength is measured rather than nightlength, with the photophase at this level of irradiance (but see text). From Saunders (1983, 1987).

described above. For this reason, this species may possess photoperiodic photoreceptors significantly less sensitive than those in insects living in more cryptic situations, such as larvae of the flesh fly, S. argyrostoma, feeding within an animal carcase. If this were true, the use of experimental photophases of higher irradiance might strengthen light pulse PTCs from Type 1 to Type 0 - as in S. argyrostoma
(Fig. 9) - and, in doing so, reset the circadian system to CT $12 \mathrm{~h}$ at the end of longer light pulses, thereby leading to nightlength measurement (as in many other species) showing evidence of external coincidence. This possibility is by no means trivial, indicating that the photoperiodic clock controlling diapause induction in P. apterus may be like many other such clocks with a photoinducible phase in the late subjective night. If, on the other hand, diapause induction were found to be regulated by the duration of the photophase, even at these higher light intensities, a photoinducible phase would of necessity occur early in the subjective night. Such a distinction might then have consequences, not only in determining whether the photoperiodic clock in $P$. apterus resembles that in other insects, but also whether operation of a specific light-sensitive or 'photoinducible' phase, occurring late in the night and sensitive to illumination at dawn, is a crucial first step in this species' photoperiodic induction.

ACKNOWLEDGEMENTS. Thanks are due to R.M.K. Saunders for help with figure production.

\section{REFERENCES}

ANDREWARTHA H.G. 1952: Diapause in relation to the ecology of insects. - Biol. Rev. 27: 50-107.

BÜNNING E. 1935: Zur kenntnis der endogenen Tagesperiodizitat bei Insekten und Pflanzen. - Ber. Dt. Bot. Ges. 53: 594-623.

BüNNING E. 1936: Die endogene Tagesrhythmik als Grundlage der photoperiodischen Reaktion. - Ber. Dt. Bot. Ges. 54: 590-607.

BüNNING E. 1960: Circadian rhythms and time measurement in photoperiodism. - Cold Spring Harb. Symp. Quant. Biol. 25: 249-256.

Chandrashekaran M.K. \& Engelmann W. 1973: Early and late subjective night phases of the Drosophila pseudoobscura circadian rhythm require different energies of blue light for phase shifting. - Z. Naturforsch. 28C: 750-753.

Chandrashekaran M.K. \& Engelmann W. 1976: Amplitude attenuation of the circadian rhythm in Drosophila pseudoobscu$r a$ with light pulses of varying irradiance and duration. - Int. J. Chronobiol. 4: 231-240.

Chandrashekeran M.K. \& Loher W. 1969: The relationship between the intensity of the light pulses and the extent of phase shifts of the circadian rhythm in the eclosion rate of Drosophila pseudoobscura. - J. Exp. Zool. 172: 147-152.

Chovnick A. (ED.) 1960: Biological Clocks. Cold Spring Harbor Symp. Quant. Biol. Vol. 25. New York, 514 pp.

Dickson R.C. 1949: Factors governing the induction of diapause in the oriental fruit moth. - Ann. Entomol. Soc. Am. 41: 511537.

Engelmann W. 1969: Phase shifting of eclosion in Drosophila pseudoobscura as a function of the energy of the light pulse. Z. Vergl. Physiol. 64: 111-117.

Garner W.W. \& Allard H.A. 1920: Effects of the relative length of the day and night and other factors of the environment on growth and reproduction in plants. - J. Agric. Res. 18: 553606.

Goto S.G. \& Numata H. 2009: Possible involvement of distinct photoreceptors in the photoperiodic induction of diapause in the flesh fly Sarcophaga similis. - J. Insect Physiol. 55: 401407. 
HodeK I. 1971: Termination of adult diapause in Pyrrhocoris apterus (Heteroptera: Pyrrhocoridae) in the field. - Entomol. Exp. Appl. 14: 212-222.

HoDEK I. 1999: Environmental regulation and some neglected aspects of insect diapause. - Entomol. Sci. 2: 533-537.

HoDeK I. 2002: Controversial aspects of diapause development. - Eur. J. Entomol. 99: 163-173.

Kalmus H. 1935: Periodizität und Autochronie (Idiochronie) als Zeitregelnde Eigenschaffen der Organismen. - Biol. Gener. 11: 93-114.

KENNY N.A.P. \& SAUNDERS D.S. 1991: Adult locomotor rhythmicity as "hands" of the maternal photoperiodic clock regulating larval diapause in the blowfly, Calliphora vicina. - J. Biol. Rhythms 6: 217-233.

KogUre M. 1933: The influence of light and temperature on certain characters of the silkworm, Bombyx mori. - J. Dept. Agr. Kyushu Univ. 4: 1-93.

Kotwica-Rolinska J., Pivarciova L., Vaneckova H. \& Dolezel D. 2017: The role of circadian clock genes in the photoperiodic timer of the linden bug Pyrrhocoris apterus during the nymphal stage. - Physiol. Entomol. 42: 266-273.

Lankinen P., Kastally C. \& Hoikkala A. 2021: Nanda-Hamner curves show huge latitudinal variation but no circadian components in Drosophila montana photoperiodism. - J. Biol. Rhythms 20: 1-13.

LEES A.D. 1965: Is there a circadian component in the Megoura photoperiodic clock? In Aschoff J. (ed.): Circadian Clocks. North-Holland, Amsterdam, pp. 351-356.

LeEs A.D. 1973: Photoperiodic time measurement in the aphid Megoura viciae. - J. Insect Physiol. 19: 2279-2316.

Marcovitch S. 1923: Plant lice and light exposure. - Science 58: $537-538$.

Marcovitch S. 1924: The migration of the Aphididae and the appearance of sexual forms as affected by the relative length of daily light exposure. - J. Agric. Res. 27: 513-522.

Mukai A., Yamaguchi K. \& Goto S.G. 2021: Urban warming and artificial light alter dormancy in the flesh fly. $-R$. Soc. Open Sci. 8: $210866,10 \mathrm{pp}$.

NANDA K.K. \& HAMNER K.C. 1958: Studies on the nature of the endogenous rhythm affecting photoperiodic response of Biloxi soy bean. - Bot. Gaz. 120: 14-25.

Norris K.H., Howell F., Hayes D.K., Adler V.E., Sullivan W.N. \& SCHECHTER M.S. 1969: The action spectrum for breaking diapause in the codling moth, Laspeyresia pomonella (L.), and the oak silkworm, Antheraea pernyi Guer. - Proc. Natl. Acad. Sci. USA 63: 1120-1127.

Paris O.H. \& JenNer C.E. 1959: Photoperiodic control of diapause in the pitcher-plant midge, Metriocnemus knabi. In Withrow R.B. (ed.): Photoperiodism and Related Phenomena in Plants and Animals. Am. Ass. Adv. Sci., Washington, pp. 601-624.

PitTENDRIGH C.S. 1954: On temperature independence in the clock controlling emergence time in Drosophila. - Proc. Natn. Acad. Sci. USA 40: 1018-1029.

Pittendrigh C.S. 1960: Circadian rhythms and the circadian organization of living systems. - Cold Spring Harb. Symp. Quant. Biol. 25: 159-184.

Pittendrigh C.S. 1966: The circadian oscillation in Drosophila pseudoobscura pupae: a model for the photoperiodic clock. Z. Pflanzenphysiol. 54: 275-307.
PitTENDRIGH C.S. 1972: Circadian surfaces and the diversity of possible roles of circadian organization in photoperiodic induction. - Proc. Natn. Acad. Sci. USA 69: 2734-2717.

SABrosky C.W., LARSON I. \& Nabours R.K. 1933: Experiments with light upon reproduction, growth and diapause in grouse locusts. - Trans. Kansas Acad. Sci. 36: 298-300.

SAUNDERS D.S. 1971: The temperature-compensated photoperiodic clock 'programming' development and pupal diapause in the flesh-fly, Sarcophaga argyrostoma. - J. Insect Physiol. 17: 801-812.

SAUNDERS D.S. 1973: The photoperiodic clock in the flesh-fly, Sarcophaga argyrostoma. - J. Insect Physiol. 19: 1941-1954.

SAUNDERS D.S. 1978: An experimental and theoretical analysis of photoperiodic induction in the flesh-fly Sarcophaga argyrostoma. - J. Comp. Physiol. (A) 124: 75-95.

SAUNDERS D.S. 1979: External coincidence and the photoinducible phase in the Sarcophaga photoperiodic clock. - J. Comp. Physiol. (A) 132: 179-189.

SAUNDERS D.S. 1982: The effect of ultra-short photoperiods on the seasonal clock in Sarcophaga argyrostoma. - J. Comp. Physiol. (A) 145: 421-429.

SAUNDERS D.S. 1983: A diapause induction-termination asymmetry in the photoperiodic responses of the linden bug, Pyrrhocoris apterus, and an effect of near-critical photoperiods on development. - J. Insect Physiol. 29: 399-405.

SAUNDERS D.S. 1987: Insect photoperiodism: the linden bug, Pyrrhocoris apterus, a species that measures daylength rather than nightlength. - Experientia 43: 935-937.

SAUNDERS D.S. 2013: Insect photoperiodism: measuring the night. - J. Insect Physiol. 59: 1-10.

SAUNDERS D.S. 2020: Dormancy, diapause, and the role of the circadian system in insect photoperiodism. - Annu. Rev. Entomol. 65: 19.1-19.17.

SAUNDERS D.S. 2021a: Insect photoperiodism: Bünning's hypothesis, the history and development of an idea. - Eur. J. Entomol. 118: 1-13.

SAUNDERS D.S. 2021b: A comparative study of circadian rhythmicity and photoperiodism in closely related species of blow flies: external coincidence, maternal induction and diapause at northern latitudes. - J. Biol. Rhythms 36: 532-547.

TeEts N.M. \& Meutr M.E. 2021: Hello darkness, my old friend: a tutorial of Nanda-Hamner protocols. - J. Biol. Rhythms 36: 221-225.

Vaz Nunes M. \& Saunders D.S. 1999: Photoperiodic time measurement in insects: a review of clock models. $-J$. Biol. Rhythms 14: 84-104.

Vaze K.M. \& Helfrich-Forster C. 2016: Drosophila ezoana uses an hour-glass or highly damped circadian clock for measuring night length and inducing diapause. - Physiol. Entomol. 41: $378-389$.

WAY M.J. \& HopkINS B.A. 1950: The influence of photoperiod and temperature on the induction of diapause in Diataraxia oleracea L. - J. Exp. Biol. 27: 365-376.

WINFREE A.T. 1970: Integrated view of resetting a circadian clock. — J. Theor. Biol. 28: 327-374.

WINFreE A.T. 1974: Suppressing Drosophila's circadian rhythm with dim light. - Science 183: 970-972.

Winfree A.T. 1980: The Geometry of Biological Time. Springer, New York, Heidelberg, Berlin, 530 pp.

Received December 5, 2021; revised and accepted January 13, 2022 Published online January 19, 2022 